\title{
Teens and Sexting - a Public Health concern?
}

Authors: Sally Handley ${ }^{1 *}$, Rachael Murray², Manpreet Bains ${ }^{2}$

'Nottinghamshire County Council ${ }^{2}$ University of Nottingham

\section{Background}

Sexual messaging (sexting) has become a norm of peer-to-peer communication among young people. There are concerns about the negative impact sexting has on young people's health and wellbeing. However, little is known about the nature of public health messages currently being provided on sexting. This study sought to understand the nature of information and advice on sexting available online for children, young people and adults.

\section{Study Aim}

To explore what information and key messages about teen sexting are available from national children's agencies that promote the welfare of children and young people.

The definition of sexting applied to the study:

'An exchange between young people of sexual messages or images of a sexual nature through mobile phones and/or the internet (with a focus on young people aged 18 and under).'

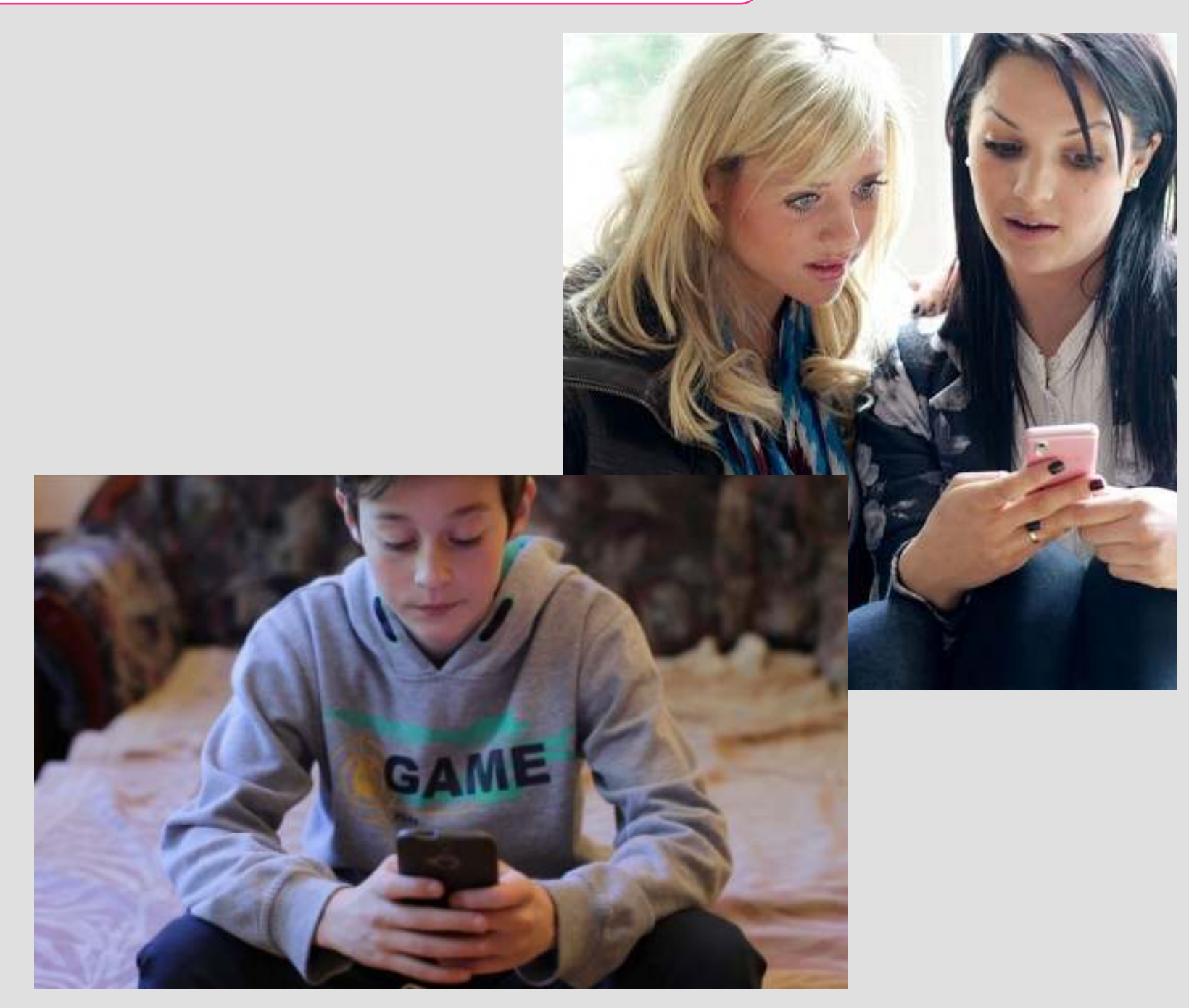

\section{Method}

A document analysis of online resources and literature available on the world wide web, from national agencies that promote the welfare of children and young people. An assessment of the target audience of the data and the review of the nature and consistency of advice available about teen sexting. An assessment of the advice presented as to whether it was cognisant with normative healthy sexual development that supported young peoples sexual agency.

The study applied a purposive, literature sampling strategy. The inclusion criteria for the data reflected the project definition of sexting. To ensure the data had relevance to the study period publication dates for inclusion were 01.01.2007 - 01.04.2015.

Data formats included: online, web based, paper based (PDF), audio visual and YouTube. Qualitative data analysis was undertaken, applying an inductive approach to the thematic analysis' of data, using QSR Nvivo qualitative data analysis software ( $\mathrm{vl0}$ for Mac). Ethics for the study were considered by the University of Nottingham Ethics Subcommittee and permission was not required.

\section{Findings}

75 data sources were considered for inclusion, of which 38 (50.5\%) met the inclusion criteria. Four themes emerged:

I Information about teen sexting 2 Safeguarding and legal issues 3 Consequences and impact 4 Advice

\section{Table I-Summary of the focus of data in each theme}

The nature of advice focused on how to say 'no', minimising risk, safety and harm reduction (including online safety), dealing with the problem, relationship advice, how to use social media, legal issues, consequences and signposting.

The target audience of the data were children/young people (26\%), parents/ carers $(39 \%)$ and teachers/professionals (35\%), with some data targeted to more than one audience.

There was a large volume of data in each theme, summary of the focus of data in each theme is shown in Table I.
Theme I Information about impact of teen sexting, the immediacy of technology, perceptions about teen sexting which varied from being 'shocking' to the normalisation of peer-to-peer sexting within young people's relationships. A resource for teachers explored; betrayal, peer pressure, low self esteem. Data for parents explored the extent of the problem and concerns about the loss of control.

Theme 2 Safeguarding advice (63\%) and information about the legal framework in the UK (74\%), where the sharing of sexually explicit images of under $18 \mathrm{~s}$ is illegal. It was clear in the data that the welfare of the child or young person was seen as paramount.

Theme $3 \mathbf{7 9 \%}$ of data considered immediate and longer term consequences. Data explored reputational damage, psycho-social distress and the negative impact on well-being when things go wrong. An agency advised that 'the effects of others seeing this content can lead to negative comments and bullying, and may result in a young person losing confidence or self esteem, and in extreme circumstances can lead to depression and other risks'.

Theme 4 Data primarily focused on minimising harm (84\%) including preventative advice, advice about online safety, online relationships and the safe use of social media (63\%). The study identified 10 of 38 data $(26 \%)$ where the advice promoted young people's sexual agency. Signposting, with a focus on safety and harm reduction, support and advice was found in $71 \%$ of data.

\section{Discussion and recommendations}

Peer-to-peer sexting may play an important part in normative sexual development and sexual enquiry². There is evidence that online digital relationships create concern for some children and young people, particularly when there is distortion of power, coercion, control or revenge ${ }^{2,3}$. Emerging research suggests that some children may present with characteristics that increase their vulnerability to the associated risk and harm from sexting ${ }^{3}$. This study found that there was a wealth of information and advice available about teen sexting with the nature of advice consistent across agencies, with agencies freely signposting to other agencies.

Harm reduction could be strengthened through a multi-agency commitment to promote inclusive, cross-curricular online safety and healthy peer relationship messages that promote young people's health, wellbeing and sexual agency. 